\title{
Effect of a Tablet Containing Probiotic Bacteria and Stomachic Herbs on Human Fecal Microbiota
}

\author{
Yasunori YONEJIMA ${ }^{1,2 *}$, Yoshiro MORI ${ }^{1}$ and Kazunari USHIDA ${ }^{2}$ \\ 1 Research and Development Department, Nitto Pharmaceutical Industries, Ltd., 35-3 Minamibiraki, Kamiueno-cho, Muko 617-0006, \\ Japan \\ 2 Laboratory of Animal Science, Kyoto Prefectural University, Shimogamo, Kyoto 606-8522, Japan
}

Received May 1, 2008; Accepted June 26, 2008

\begin{abstract}
We evaluated the effects of a tablet containing Bacillus natto and Lactobacillus acidophilus as probiotics supplemented with three stomachic herbs on human fecal microbiota. Six healthy subjects ingested 3 tablets, 3 times a day after meals for 10 days. Fecal samples were collected before and after the administration period, and 1 week post administration. As shown by the TGGE images, the density of the bands identified with Bifidobacterium increased in four cases. This increase was confirmed by real-time PCR. It was observed that the densities of the bands identified with Haemophilus decreased, Ruminococcus decreased, Clostridium colinum decreased, Acidaminococcus increased and Megamonas increased individually.
\end{abstract}

Key words: Bacillus natto; Lactobacillus acidophilus; stomachic herbs; human intestinal microflora

Probiotic bacteria, namely lactobacilli and bifidobacteria, are now well recognized for their health promoting effects (5). Administration of probiotics is often realized orally by a single dose, although sometimes they are dosed with medicinal plant materials.

Lactic acid bacteria react with gut epithelial tissues to affect the mucosal immunity through the stimulation of toll-like receptors (TLRs). Lactic acid bacteria affect the host physiology through chloride secretion from the gut, to increase water contents of the digesta (1), and modification of the sympathetic and parasympathetic nervous systems, to reduce blood pressure and blood sugar levels (13). Probiotics also strongly affect the host's indigenous intestinal microbiota $(9,10)$. Indeed, they promote different colonic fermentation patterns (11). Probiotics, more precisely fermented milk, may affect the gastric emptying, reducing the digesta transit (12). Digesta kinetics have strong influences on the digestion, therefore the administration of probiotics can modulate the amount of material flowing into the large intestine. The availability of substrate should modify the composition of the microbiota.

Medicinal plants such as swertia, cinnamon, and fennel, have long been administered to patients with digestive troubles. They engage with the host's digestive physiological systems, though the mechanisms are undefined. The introduction of these medicinal plants into probiotic therapies may modulate the effects of

*Corresponding author. Mailing address: Research and Development Department, Nitto Pharmaceutical Industries, Ltd., 35-3 Minamibiraki, Kamiueno-cho, Muko 617-0006, Japan. Phone: +81-75-921-5344. Fax: +81-75-934-4889. E-mail: y.yonejima@nitto-ph.com probiotics.

In this report, we show the effect of a tablet containing both probiotic and medicinal herbs on the fecal microbiota in human volunteers.

Six healthy volunteers ( 4 men and 2 women, aged from 24 to 55 years old, average age: 36 ) were recruited from Nitto Pharmaceutical Industries, Ltd. to test a tablet containing probiotics and medicinal plants. Experiments were conducted in accordance with the Declaration of Helsinki. All subjects were informed of the details of the present study and gave their consent to participate. No restrictions of food choice were imposed on the subjects throughout the study, except prohibition of ingestion of fermented milk, fermented soybeans and pickles the day before the sampling.

The tablet is a probiotic product, the Guard (Kowa Shinyaku Co., Ltd. Japan) and contains Bacillus natto NT (Bacillus natto $)\left(10^{8} \mathrm{cfu} / 9\right.$ tablets), Lactobacillus acidophilus NT (Lactomin) ( $10^{9} \mathrm{cfu} / 9$ tablets), dimethylpolysiloxane, powdered swertia herb, powdered cinnamon bark, powdered fennel, methyl methionine sulfonium chloride, precipitated calcium carbonate, magnesium carbonate (Table 1).

The 6 healthy subjects took 3 tablets, 3 times a day after meals for 10 days. Stool samples were collected before and after the administration period. An additional sampling was made 7 days after the end of administration.

Fresh feces were transferred into sterile plastic tubes $(50 \mathrm{ml})$ containing $30 \mathrm{ml}$ of ethanol to maintain an ethanol concentration in excess of $70 \%$. Samples of feces were kept at $5{ }^{\circ} \mathrm{C}$ until the analyses, which were 
Table 1. Ingredients of the tablet

\begin{tabular}{lr}
\hline Ingredient & dosage \\
\hline Bacillus natto & $10 \mathrm{mg}$ \\
Lactomin & $30 \mathrm{mg}$ \\
Dimethylpolysiloxane & $84.6 \mathrm{mg}$ \\
Powdered Swertia Herb & $30 \mathrm{mg}$ \\
Powdered Cinnamon Bark & $30 \mathrm{mg}$ \\
Powdered Fennel & $30 \mathrm{mg}$ \\
Methyl Methionine Sulfonium Chloride & $30 \mathrm{mg}$ \\
Precipitated Calcium Carbonate & $300 \mathrm{mg}$ \\
Magnesium Carbonate & $300 \mathrm{mg}$ \\
\hline
\end{tabular}

performed within three months of collection. After homogenization, a portion of the suspension was centrifuged $\left(6,700 \times \mathrm{g}\right.$, for $\left.10 \mathrm{~min}, 4^{\circ} \mathrm{C}\right)$ and the pellets were collected. Approximately $0.2 \mathrm{~g}$ of pellets were washed twice with $1 \times$ phosphate-buffered saline (PBS). Bacterial DNA was extracted from the pellets using ISOFECAL for Beads Beating kit (NIPPON GENE CO., LTD.) and FastPrep FP120 (BIO101, Inc.).

All individual samples of bacterial 16S rDNA were subjected to temperature gradient gel electrophoresis (TGGE) analysis as described elsewhere $(2,8)$.

The gel image was taken by a densitometer (GS-800; Bio-Rad, Tokyo, Japan). The profiles were analyzed using a software package for imaging and analyzing electrophoresis gels (Quantity one, Bio-Rad, Tokyo, Japan), and cluster analysis of the TGGE band profiles was performed using hierarchical clustering analysis with Euclidean square distances using the Microsoft Excel macro program developed by S. Aoki (Gunma University, Maebashi, Japan; program available at http:// aoki2.si.gunma-u.ac.jp/lecture/stats-by-excel/vba/html/ clustan.htm). A corresponding dendrogram was constructed with Ward linkages. In this study, very weakly stained bands with an optical density (OD) of $<0.1$ as determined by the software were ignored, because on occasion such bands cannot be identified automatically by the software and this could result in artifacts in the analysis. The density of the bands, as determined by the software, was not used in the analysis. If a lane had a band at a certain position, an assignment of " 1 " was made; if there was no band at the same position, an assignment of " 0 " was made. A mixture of $16 \mathrm{~S}$ rDNA of known bacteria (2) was applied to the TGGE gels as standard markers. B.natto NT and L. acidophilus NT were also used as standards.

Twenty one bands on the TGGE gel were excised and put into $20 \mu \mathrm{l}$ of sterile water. The excised gel was left in water overnight at $4{ }^{\circ} \mathrm{C}$ to elute the DNA (11). These bands (indicated in Fig. 3) were selected because their densities had changed remarkably between before and after the probiotic administration. After the extraction step was performed, a portion of eluted DNA solution was amplified with primers $968 \mathrm{~F}$, which do not contain the 40 bases GC clump and univ-R. The PCR conditions were principally the same as described elsewhere (2) except for the cycle number: 30 cycles.

The size and amount of the PCR products were confirmed by analyzing all samples by $1.5 \%$ agarose gel (wt/vol) electrophoresis and ethidium bromide staining. The obtained bands were purified using Wizard SV Gel and PCR Clean-UP System (Promega, Tokyo, Japan).

Purified products were sequenced after TA-cloning, as described elsewhere (11). Briefly, PCR amplicons were ligated into pGEM-T vector (Bio-Rad, Tokyo, Japan) and Escherichia coli JM109 was transformed. Clones harboring amplified DNA were selected by blue-white screening, and 8 white clones were randomly selected from each plate (one plate for each TGGE band). Colony PCR using SP6 and T7 primers was performed to check the size of inserted DNA with r-Taq polymerase. The following thermal cycle was applied: $94^{\circ} \mathrm{C}$ for $3 \mathrm{~min}$ of initial denaturation followed by 25 cycles of $94^{\circ} \mathrm{C}$ for 30 sec, $48^{\circ} \mathrm{C}$ for $30 \mathrm{sec}$, and $72^{\circ} \mathrm{C}$ for $50 \mathrm{sec}$, with a final elongation at $72^{\circ} \mathrm{C}$ for $3 \mathrm{~min}$. Restriction fragment length polymorphism (RFLP) analyses of PCR products were performed with Hae III (Toyobo, Tokyo, Japan), Hha I (Toyobo), and Rsa I (Toyobo) according to the manufacturer's instructions to group the obtained E.coli clones. Although several clone groups were recognized for each excised TGGE band, the most abundant clone group was selected and the insert of one randomly selected clone from the clone group was sequenced. Sequencing was performed at Shimadzu Genomic Research, Co., Ltd. (Kyoto, Japan). Sequences were compared via the BLAST program at NCBI (http:// www.ncbi.nlm.nih.gov/blast) with those registered in databases (such as DDBJ/EMBL/ GenBank) to suggest possible taxonomic names.

The relative abundance of bifidobacterial $16 \mathrm{~S}$ rDNA in fecal samples was quantified with the Light Cycler system (Roche Diagnostics, Tokyo, Japan).The FastStart DNA Master SYBR Green I was used for PCR. The reaction mixture $(20 \mu \mathrm{l})$ contained $3 \mathrm{mM} \mathrm{MgCl} 2,2 \mu \mathrm{l}$ of the $10 \times$ Mastermix, $20 \mathrm{ng}$ of fecal DNA, and $0.5 \mu \mathrm{M}$ of each primer. For quantification of total bifidobacterial $16 \mathrm{~S}$ rDNA, the primers g-Bifid-F (5'CTCCTGGAAACGGGTGG-3') and g-Bifid-R (5'GGTGTTCTTCCCGATATCTACA-3') were used (7). The thermal program consisted of initial denaturation at 
$95^{\circ} \mathrm{C}$ for $30 \mathrm{sec}$ followed by 40 cycles of $95^{\circ} \mathrm{C}$ for $4 \mathrm{sec}$ and $62^{\circ} \mathrm{C}$ for $25 \mathrm{sec}$. Dilutions of the genomic DNA from Bifidobacterium longum JCM7050 of known amount of viable count were used to construct calibration curves. These calibration curves were applied to the calculation of the total count of Bifidobacterium in fecal samples.

The bacterial species, which may typically contribute to the change in the diversity of the intestinal microbiota, appearing as TGGE bands were detected. The detected bands are shown in Fig. 1.

The band profiles differing between before and after intake as evidenced by cluster analysis are shown in Fig. 2. According to the dendrogram created from the TGGE band profiles, profiles from D-after and E-after constructed one cluster. Others tended to construct one cluster for each subject such as A-before with A-after. According to the clustering analysis (Fig. 2), Subjects A, $\mathrm{B}, \mathrm{C}$ and $\mathrm{F}$ formed one group, and subject $\mathrm{D}$ clustered with subject $\mathrm{E}$. This result indicates that the intestinal microbiota of subjects D and E were relatively similar to each other and that the composition of microbiota was modified in a similar manner by the mixture of probiotics and herbs.

There was a band with increased density which was commonly shared by 4 subjects (A, D, E and F) below the bottom markers. On the other hand, there were no remarkable changes in the bands which existed at the same position in lane $L$. acidophilus $\mathrm{NT}$ and B. natto NT for each subject. Twenty-one TGGE bands, indicated in Fig. 3, whose density changed remarkably were identified. Table 2 shows the results of sequence analyses of these TGGE bands. Bifidobacterium-like sequences were determined for band 1 to band 10; Haemophiluslike sequences were determined for band 11 and band 12; a butyrate-producing bacterium-like sequence was determined for band 13; an Acidaminococcus-like sequence was determined for band 14; Ruminococcuslike sequences were determined for band 15 and band 16; a butyrate-producing bacterium-like sequence was determined for band 17; a Clostridium colinum-like sequence was determined for band 18; a Ruminococcuslike sequence and a Clostridium orbiscidens-like sequence were determined for band 19; and Megamonaslike sequences were determined for band 20 and band 21 .

In the case of subject $A$, the density of the bands identified as Bifidobacterium increased after the probiotic tablet administration. In the case of subject B, the density of the bands identified as Haemophilus and butyrate-producing bacterium decreased and the density of the band identified as Acidaminococcus increased after the administration. In the case of subject $C$, the

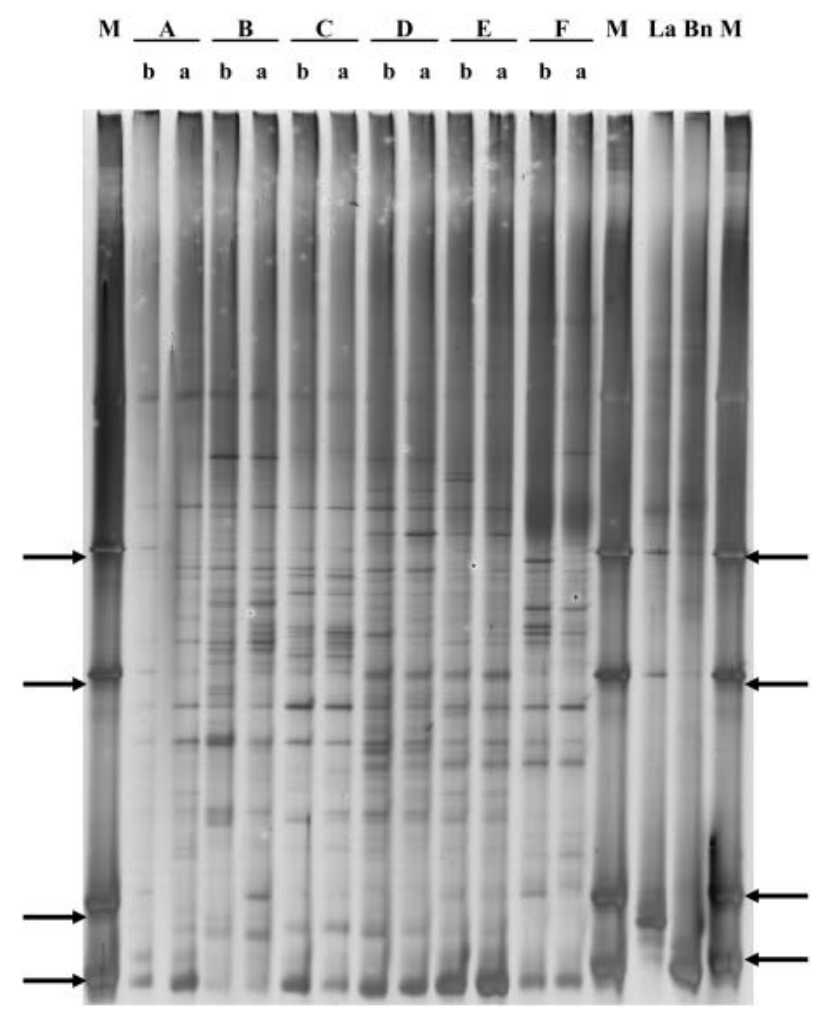

Fig.1. Gel image of TGGE of bacterial 16S rDNA (developed by silver staining). A-F: Subjects; La: Lactobacillus acidophilus NT 16S rDNA gene; Bn: Bacillus natto NT 16S rDNA gene; M: marker; b: Before administration; a: After administration. The markers consisted of four bacterial 16S rDNA genes prepared in our laboratory. The four horizontal arrows along with the standard marker lanes indicate the 16S rDNA gene bands resembling those of Ruminococcus hydrogenotrophicus (Accession number of GenBank: X95624, homology 91\%), Clostridium sp. (AF157053, homology 85\%), Escherichia coli (A14565, homology 100\%), and Lactobacillus gasseri strain KC5a (AF243165, homology 97\%) from top to bottom.

density of the bands identified as Ruminococcus and Bifidobacterium decreased after the administration. In the case of subject $\mathrm{D}$, the density of the bands identified as butyrate-producing bacterium and Bifidobacterium increased after the administration. In the case of subject $\mathrm{E}$, the density of the bands identified as Bifidobacterium increased after the administration. In the case of subject $\mathrm{F}$, the density of the bands identified as Bifidobacterium, Megamonas and Ruminococcus or Clostridium orbiscidens increased and the density of the band identified as Clostridium colinum decreased after the administration.

The estimated total cell numbers of bifidobacteria are listed in Table 3. The number of bifidobacteria increased after administration of the probiotic tablet in four subjects (A, D, E and F), decreased a little in one subject 
Euclid squarc distance

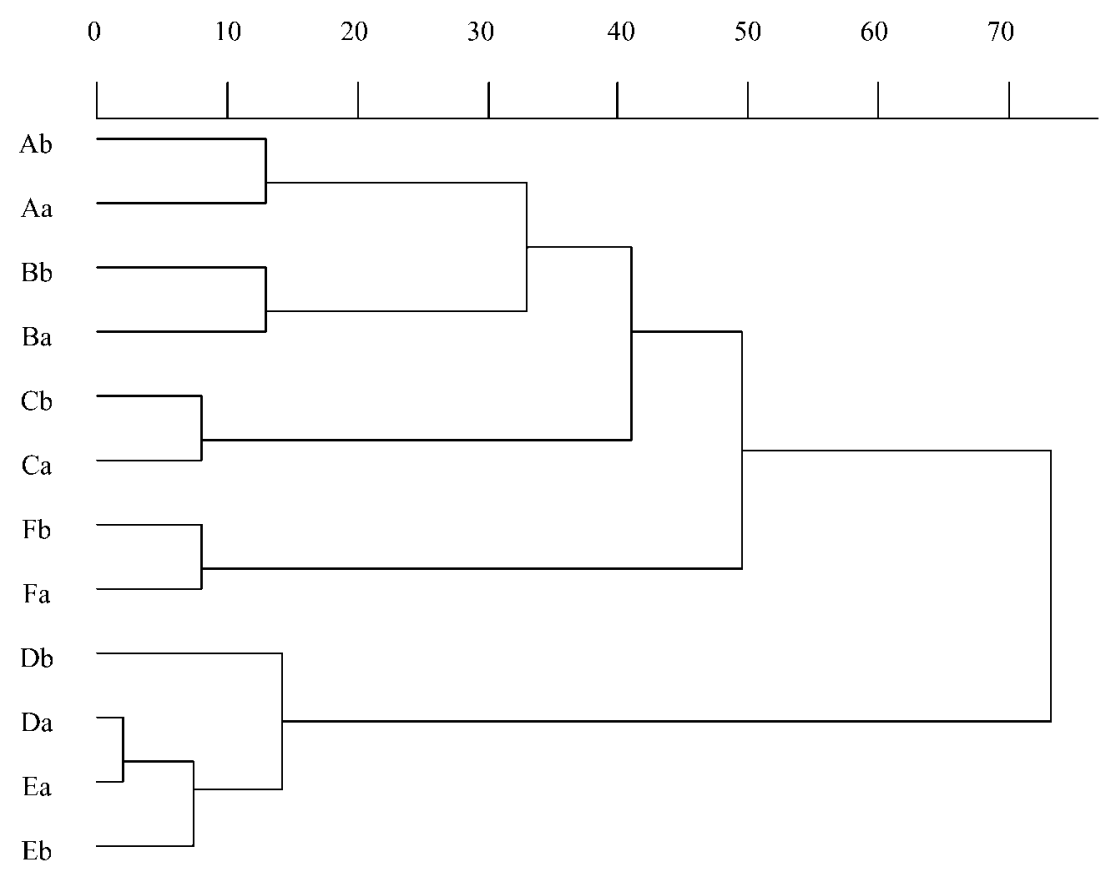

Fig.2. Dendrogram obtained from hierarchical clustering of the TGGE band profiles. A-F: Subjects; b: Before administration; a: After administration.

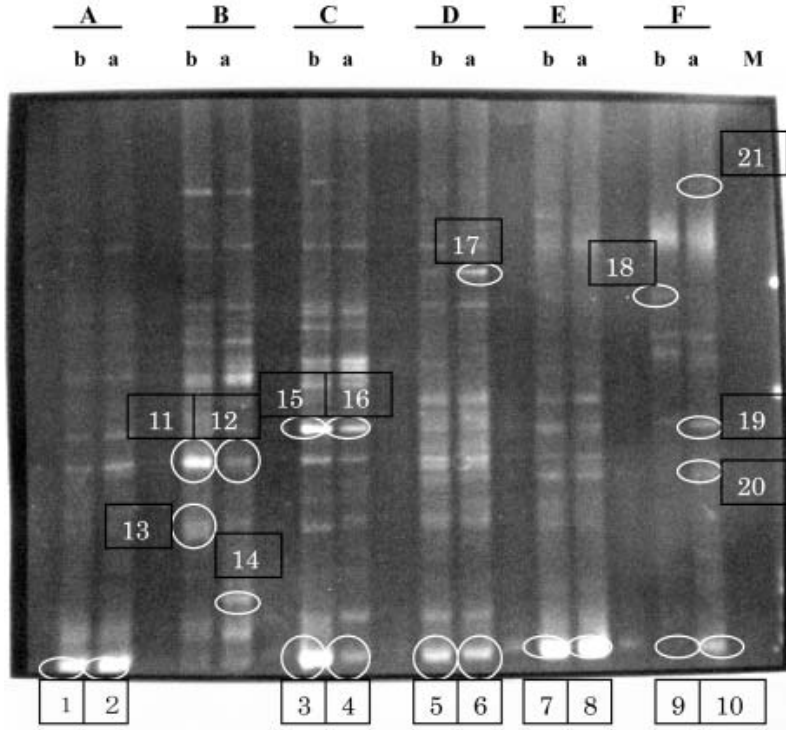

Fig.3. Gel image of TGGE of bacterial 16S rDNA (developed by ethidium bromide staining). A-F: Subjects; M: marker; b: Before administration; a: After administration.
(C), and decreased in one subject (B).The TGGE results showed common and individual changes of intestinal microflora among the six subjects. In four cases out of six, the density of the bands identified with Bifidobacterium increased after administration of the probiotic tablet. This was confirmed by real-time PCR. The results indicate that intake of the probiotic tablet affected colonic environment supporting the growth of Bifidobacterium. The three stomachic herbs would have promoted digestion and changed the composition of the material flowing into the intestine. The increase in Bifidobacterium was considered to be due to the effect of the herbs. However, there was no significant difference between the "Before" and the "After" samples (Table 3), because two of the six subjects (B and C) showed an inverse tendency in population size of bifidobacteria. The results indicate that the probiotic tablet had a bifidogenic effect on at least four of the six subjects with different magnitudes. Individual differences in response to the tablet administration may reflect individual differences in composition of intestinal microflora. A similar observation was reported by Ohashi et al. (11) about the cecal fermentation pattern.

In the present experiment, for example, subjects D and 
Table 2. Results of BLAST searches for partial sequences of bacterial 16S rDNA

\begin{tabular}{|c|c|c|c|}
\hline Subjects & Band No. & First match with known bacterium (Accession No.) & Identity $(\%)$ \\
\hline \multirow[t]{4}{*}{ A } & 1 & Bifidobacterium sp. (AF306789) & 100 \\
\hline & & Collinsella aerofaciens (AJ245920) & 99 \\
\hline & 2 & Bifidobacterium longum (EF589112) & 100 \\
\hline & & Lactobacillus sp. (AB158766) & 96 \\
\hline \multirow[t]{4}{*}{ B } & 11 & Haemophilus parainfluenzae (EU083530) & 99 \\
\hline & 12 & Haemophilus parainfluenzae (EU083530) & 99 \\
\hline & 13 & Butyrate-producing bacterium (AJ270469) & 100 \\
\hline & 14 & Acidaminococcaceae bacterium (AB298734) & 93 \\
\hline \multirow[t]{5}{*}{$\mathrm{C}$} & 3 & Bifidobacterium sp. (AF306789) & 99 \\
\hline & 4 & Bifidobacterium sp. (AF306789) & 100 \\
\hline & & Bifidobacterium longum (EF589112) & 100 \\
\hline & 15 & Ruminococcus sp. (EF036467) & 100 \\
\hline & 16 & Ruminococcus sp. (EF036467) & 100 \\
\hline \multirow[t]{6}{*}{$\mathrm{D}$} & 5 & Bifidobacterium adolescentis (AP009256) & 98 \\
\hline & & Bifidobacterium longum (EF589112) & 99 \\
\hline & & Clostridium sp. (DQ100445) & 91 \\
\hline & 6 & Bifidobacterium adolescentis (AP009256) & 99 \\
\hline & & Collinsella aerofaciens (AJ245920) & 100 \\
\hline & 17 & Butyrate-producing bacterium (AJ27042) & 94 \\
\hline \multirow[t]{2}{*}{$\mathrm{E}$} & 7 & Bifidobacterium adolescentis (AY305304) & 99 \\
\hline & 8 & Bifidobacterium longum (EF589112) & 100 \\
\hline \multirow[t]{7}{*}{$\mathrm{F}$} & 9 & Bifidobacterium longum (EF589112) & 100 \\
\hline & 10 & Bifidobacterium longum (EF589112) & 100 \\
\hline & 18 & Clostridium colinum (X76748) & 92 \\
\hline & 19 & Ruminococcus sp. (EF036467) & 99 \\
\hline & & Clostridium orbiscidens (Y18187) & 98 \\
\hline & 20 & Megamonas sp. (EU346729) & 99 \\
\hline & 21 & Megamonas sp. (EU346729) & 97 \\
\hline
\end{tabular}

Table 3 . The relative abundance $(\log 10$ cell/g feces) of each operational taxonomic unit (OTU) related to bifidobacteria in the feces

\begin{tabular}{cccc}
\hline Subjects & Before & After & Post \\
\hline A & 8.71 & 9.28 & 10.08 \\
B & 9.97 & 8.74 & 9.57 \\
C & 9.7 & 9.28 & 9.85 \\
D & 10.38 & 10.48 & 10.29 \\
E & 10.43 & 10.57 & 10.03 \\
F & 9.75 & 9.92 & 9.38 \\
\hline
\end{tabular}

Before: Before administration; After: After administration; Post: Post-administration.

E, both had relatively similar intestinal microflora as evidenced by the cluster analysis of their TGGE profiles. Bifidobacteria increased by some 30 to $40 \%$ after administration of the probiotic tablet. Subject F showed a similar level of increase (45\%) in the size of the bifidobacterial population. Among the four positively responding subjects in terms of bifidobacteria, subject $\mathrm{A}$ showed a notable increase of $380 \%$. Interestingly, subject A showed the smallest bifidobacterial population before the administration of the tablets. At the moment we do not know of any specific component of intestinal microflora other than bifidobacteria which would result in an increase in bifidobacteria.

Another particular modification in intestinal microbiota was the stimulation of lactate-utilizing bacteria such as Acidaminococcus, butyrate-producing bacterium, and Megamonas. These bacteria produced butyric acid or propionic acid from lactic acid. An increase in bifidobacteria may help higher lactate production, but it is important to convert lactate into short-chain fatty acids by lactate-utilizers, because butyrate and propionate have particular importance for the host health $(3,4)$.

This type of human study needs a certain level of subject numbers due to the tremendous heterogenesity of human intestinal microbiota. Indeed, each person has his 
(her) own composition of microbiota. However, even with the limited number of subjects, there was no general tendency of modification of intestinal bacteria. In this experiment, since only 6 subjects were available, the sample number was too small to draw a definitive conclusion. Individual differences in intestinal microbiota may be large enough to react with probiotics differently, because the interaction between indigenous intestinal bacteria and probiotic strains differ from one individual to another $(10,11)$. In the case of rodent models, the effects of intestinal modifiers such as dietary prebiotics show a defined tendency as evidenced by clearly separated clusters (i.e., administer and nonadminiser) (6). This is because the rodent model has a relatively well defined and simplified intestinal microbiota (14). Such a condition cannot be achieved in human experiments. Therefore, we need substantial numbers of subjects to draw a general conclusion about the effects of probiotics or prebiotics in human cases. This experiment was indeed a preliminary one. However, stimulation of bifidobacteria was detected in four cases out of six. This can be considered as an indication of the potential of this mixture of probiotics and medicinal herb plants.

\section{REFERENCES}

(1) Inoue R, Tsukahara T, Noda M, Bukawa W, Matsubara N, Ushida K. 2007. Oral administration of a heat-killed cell preparation of Enterococcus faecalis strain EC-12 stimulates the gene expression of $\mathrm{Na}^{+}-\mathrm{K}^{+}-2 \mathrm{Cl}^{-}$cotransporter 1 in rat ileal epithelial cells. Biosci Biotechnol Biochem 71: 807-810.

(2) Inoue R, Ushida K. 2003. Development of the intestinal microbiota in rats and its possible interactions with the evolution of the luminal $\operatorname{IgA}$ in the intestine. FEMS Microbiol Ecol 45: 147-153.

(3) Kameue C, Tsukahara T, Ushida K. 2006. Alteraion of gene expression in the colon of colorectal cancer model rat by dietary sodium gluconate. Biosci Biotechnol Biochem 70: 606-614.

(4) Kishimoto A, Ushida K, Phillips GO, Ogasawara T, Sasaki Y. 2006. Identification of intestinal bacteria responsible for fermentation of gum arabic in pig model. Curr Microbiol 53: 173-177.
(5) Macfarlane GT, Cummings JH. 1999. Probiotics and prebiotics: can regulating the activities of intestinal bacteria benefit health? BMJ 318: 999-1003.

(6) Maekawa M, Ushida K. 2005. Butyrate and propionate production from d-mannitol in the large intestine of pig and rat. Microb Ecol Health Dis 17: 169-176.

(7) Matsuki T, Watanebe K, Tanaka R, Oyaizu H. 1998. Rapid identification of human intestinal bifidobacteria by $16 \mathrm{~S}$ rRNA-targeted speicies-and group-specific primers. FEMS Microbiol Lett 67: 113-121.

(8) Muyzer G, Waal EC, Uitterlinden AG. 1993. Profiling of complex microbial populations by denaturing gradient gel electrophoresis analysis of polymerase chain reaction-amplified genes coding for $16 \mathrm{~S}$ rRNA. Appl Environ Microbiol 59: 695-700.

(9) Ohashi Y, Inoue R, Tanaka M, Matsuki T, Umesaki Y, Ushida K. 2001. Lactobacillus casei strain Shirotafermented milk stimulates indigenous lactobacilli in the pig intestine. J Nutr Sci Vitaminol 47: 172-176.

(10) Ohashi Y, Tokunaga M, Taketomo N, Ushida K. 2006. Stimulation of indigenous lactobacilli by fermented milk prepared with probiotic bacterium, Lactobacillus delbrueckii subsp. bulgaricus strain 2038, in the pigs. J Nutr Sci Vitaminol 53: 82-86.

(11) Ohashi Y, Tokunaga M, Ushida K. 2004. The effect of Lactobacillus casei strain Shirota on the cecal fermentation pattern depends on the individual cecal microflora in pigs. J Nutr Sci Vitaminol 50: 399-403.

(12) Ohashi Y, Umesaki Y. 1997. Kinetics of L. casei strain Shirota fermented milk in upper gastrointestinal tract of the rat model. Dig Absorp 20: 119-123.

(13) Tanida M, Yamato T, Maeda K, Okumura N, Fukushima Y, Nagai K. 2005. Effects of intraduodenal injection of Lactobacillus johnsonii La1 on renal sympathetics nerve activity and blood pressure in urethane-anesthetized rats. Neurosci Let 389: 109114.

(14) Yanabe M, Shibuya M, Gonda T, Asai H, Tanaka T, Sudou K, Narita T, Itoh K. 2001. Establishment of specific pathogen-free (SPF) rat colonies using gnotobiotic techniques. Exp Anim 50: 293-298.

(15) Zoetendal EG, Akkermans AD, De Vos WM. 1998. Temperature gradient gel electrophoresis analysis of 16S rRNA from human fecal samples reveals stable and host-specific communities of active bacteria. Appl Environ Microbiol 64: 3854-3859. 